\title{
High-q microring resonator with narrow free spectral range for pulse repetition rate
} multiplication

Pu, Minhao; Ji, Hua; Frandsen, Lars Hagedorn; Galili, Michael; Oxenløwe, Leif Katsuo; Hvam, Jørn Märcher

Published in:

Conference proceedings, CLEO/IQEC

Publication date:

2009

Document Version

Publisher's PDF, also known as Version of record

Link back to DTU Orbit

Citation (APA):

Pu, M., Ji, H., Frandsen, L. H., Galili, M., Oxenløwe, L. K., \& Hvam, J. M. (2009). High-q microring resonator with narrow free spectral range for pulse repetition rate multiplication. In Conference proceedings, CLEO/IQEC (pp. CThBB7). IEEE.

\section{General rights}

Copyright and moral rights for the publications made accessible in the public portal are retained by the authors and/or other copyright owners and it is a condition of accessing publications that users recognise and abide by the legal requirements associated with these rights.

- Users may download and print one copy of any publication from the public portal for the purpose of private study or research.

- You may not further distribute the material or use it for any profit-making activity or commercial gain

- You may freely distribute the URL identifying the publication in the public portal

If you believe that this document breaches copyright please contact us providing details, and we will remove access to the work immediately and investigate your claim 


\title{
High-Q Microring Resonator with Narrow Free Spectral Range for Pulse Repetition Rate Multiplication
}

\author{
Minhao Pu, Hua Ji, Lars H. Frandsen, Michael Galili, Leif K. Oxenløwe, Jørn M. Hvam \\ DTU Fotonik, Department of Photonics Engineering, Technical University of Denmark, DK-2800 Lyngby, Denmark \\ mipu@fotonik.dtu.dk
}

\begin{abstract}
We demonstrate a silicon-on-insulator microring resonator with a free-spectral-range of $0.32 \mathrm{~nm}$, an extinction ratio of $27 \mathrm{~dB}$, and a quality factor of $\sim 140900$ at $1550 \mathrm{~nm}$ that is used for pulse repetition-rate multiplication from 10 to $40 \mathrm{GHz}$. (C)2009 Optical Society of America

OCIS codes: (130.3120) Integrated optics devices; (230.5750) Resonators; (120,7370) Filters;
\end{abstract}

\section{Introduction}

Integrated optical microring resonators can be applied to achieve a broad range of on-chip functionalities such as filtering, modulating, switching, etc. [1]. Recently, the spectrally periodic filtering characteristics of microrings have received increasing interests for pulse repetition-rate multiplication (PRRM) in optical communication systems [2, 3]. Fabry-Perot filters [4] and fiber Bragg gratings [5] have already been utilized for realizing PRRM. However, these devices are bulky, sensitive to ambient conditions, and not suitable for on-chip integration. In this paper, a microring resonator fabricated in silicon-on-insulator (SOI) material using CMOS compatible technologies is used for the first time to obtain PRRM of an optical $10 \mathrm{GHz}$ pulse train to $40 \mathrm{GHz}$. This device is easily integrated with photonic and electronic circuits and offers a stable filtering characteristic.

\section{Design and fabrication}

The microring resonator is designed to have a nominal free-spectral-range (FSR) of 40GHz. This requires the bending radius of the silicon ring to be $250 \mu \mathrm{m}$. The ring is directionally coupled to two waveguides guiding light to and from the ring as shown in fig. 1(a). The cross section of the ring and the waveguides is $420 \mathrm{~nm} \times 340 \mathrm{~nm}$. The gaps between the ring and the waveguides in the coupling regions are $440 \mathrm{~nm}$ and $470 \mathrm{~nm}$ for the through port and the drop port, respectively. Figure 1(b) shows a scanning electron micrograph (SEM) image of the coupling region between the through port and the microring. The gaps in the coupling regions are designed to be wider than the waveguide widths in order to make the coupling relatively weak providing a higher extinction ratio at the drop port. The microring resonator is fabricated in SOI material having a top silicon thickness of $340 \mathrm{~nm}$ and a $1 \mu \mathrm{m}$ buried silicon dioxide. Diluted (1:1 in anisole) electron-beam resist ZEP520A was spin-coated on the wafer to create a $\sim 110$ nm-thick masking layer. The microring structure was defined in the ZEP520A layer by utilizing electron-beam lithography (JEOL JBX-9300FS). The patterns were subsequently transferred to the top silicon layer by employing inductively coupled plasma reactive ion etching. A top cladding of $\sim 1 \mu \mathrm{m}$ oxide was deposited as a last step.
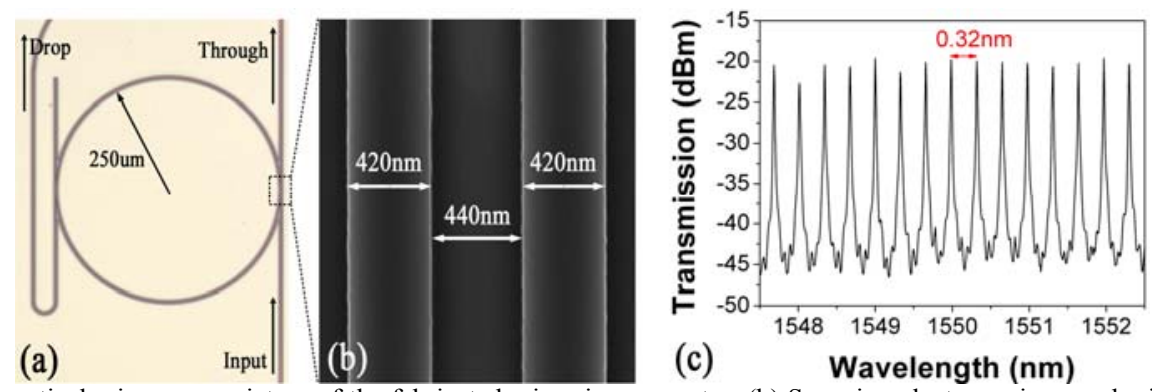

Fig. 1. (a) Top-view optical microscope picture of the fabricated microring resonator. (b) Scanning electron micrograph picture of the coupling region between the through port and the microring. (c) Transmission spectrum of the microring resonator recorded at the drop port.

A tunable (1480nm to $1580 \mathrm{~nm})$ continuous wave laser was used to record the transmission spectrum of the microring resonator. Figure 1(c) shows the output power of the quasi-transverse magnetic (TM) mode versus wavelength for the drop port. The average FSR of the fabricated microring resonator is $41.2 \mathrm{GHz}$. The extinction ratio is $\sim 27 \mathrm{~dB}$ and the $3 \mathrm{~dB}$-bandwidth is $\sim 0.011 \mathrm{~nm}$ which corresponds to a quality factor of $\sim 140900$ at $1550 \mathrm{~nm}$. At resonances, the insertion loss of the microring is $\sim 22.5 \mathrm{~dB}$ for the drop port. This includes the loss from the filter and the fiber-to-waveguide coupling losses, which are estimated to $\sim 7 \mathrm{~dB} /$ facet in the setup used. The coupling from fiber-to-chip can be improved by using e.g. nanotapered couplers. The filter loss includes the coupling losses 


\section{CThBB7.pdf}

between the waveguides and the ring and the propagation loss $\sim 0.32 \mathrm{~dB} / \mathrm{mm}$ for the quasi-TM mode. In this way, the round-trip loss of the ring is estimated to be $0.5 \mathrm{~dB}$.

\section{Experiment}

The characterization setup is schematically shown in fig. 2. The tunable mode-locked laser (TMLL) is used to generate a pulse train at a desired central wavelength from $1480 \mathrm{~nm}$ to $1580 \mathrm{~nm}$ with a repetition-rate corresponding to one quarter of the FSR of the microring. The output pulse train from the microring is monitored using an optical spectral analyzer (OSA), an electrical spectrum analyzer (ESA), and an optical sampling oscilloscope (OSC).

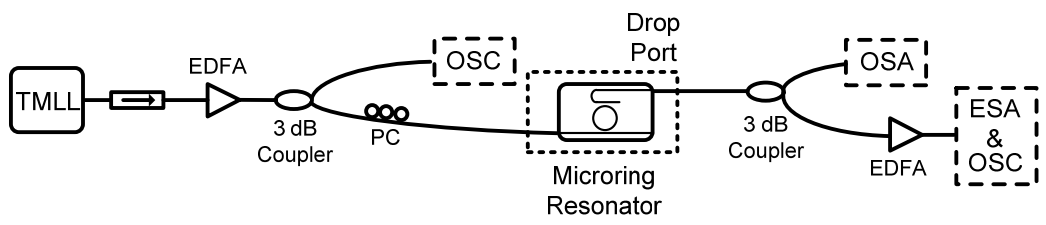

Fig.2. Experimental setup for pulse repetition rate multiplication experiment

A $10.307 \mathrm{GHz}$ pulse train (fig. 3(c)) with central wavelength $1549.56 \mathrm{~nm}$ (fig. 3(a)) is sent through the microring resonator and as shown in fig. $3(\mathrm{~d})$ a $41.2 \mathrm{GHz}$ pulse train is obtained. From figs. 3(a) and (b), we can see that most

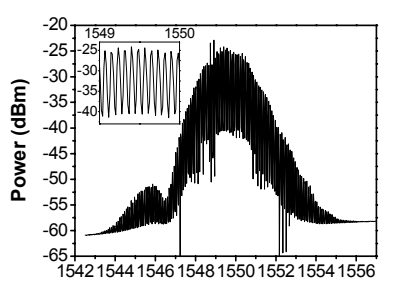

(a)

Wavelength (nm)

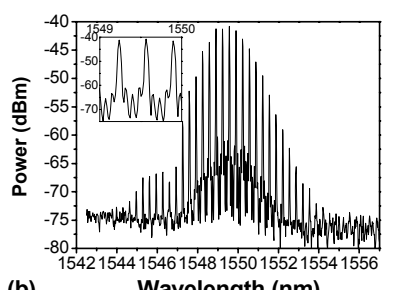

(b)

Wavelength (nm)

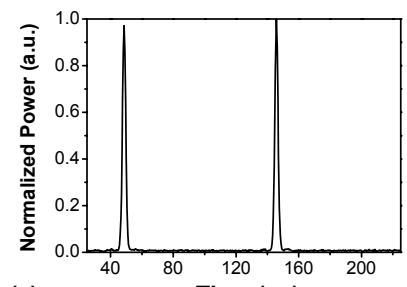

(c)

Time (ps)

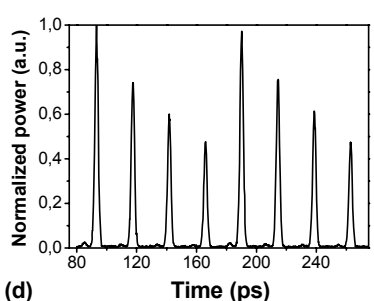

(d)

Time (ps)

, (d) $40 \mathrm{GHz}$ pulse train

of the phase-locked $40 \mathrm{GHz}$ components in the $10 \mathrm{GHz}$ comb corresponding to the input pulse train are transmitted to the drop port by the microring resonator. By measuring the electrical power spectrum, it is found that the $40 \mathrm{GHz}$ component is $21.4 \mathrm{~dB}$ stronger in amplitude than the $10 \mathrm{GHz}$ component. The original $10 \mathrm{GHz}$ pulses are $2.27 \mathrm{ps}$ (full-width half-maximum). After PRRM to $40 \mathrm{GHz}$, the pulse width is $2.45 \mathrm{ps}$ which is a broadening of less than $10 \%$. The temporal trace (fig. 3(d)) shows that the $40 \mathrm{GHz}$ pulse train has an amplitude envelope and that the pulses suffer $\sim 1 \mathrm{~dB}$ loss per round-trip in the ring. The propagation loss of the ring contributes with a loss of $0.5 \mathrm{~dB}$ and the coupling between the ring and waveguides contributes with another $0.5 \mathrm{~dB}$. The variation in pulse amplitudes can be reduced by decreasing the propagation loss by e.g. using a post thermal oxidation in the fabrication process or it can be improved by reducing the coupling between ring and waveguides, but at the expense of increased insertion loss of the filter. We have measured that the microring can tolerate up to $10 \mathrm{MHz}$ mismatch between the input repetition rate and the FSR of the filter. Clean pulse trains are obtained but the pulse width increases to 3.28 ps by detuning the input repetition rate $10 \mathrm{MHz}$ from the optimum match to the FSR. The pulses are broadened due to suboptimum filtering of the $10 \mathrm{GHz}$ modes resulting in a narrowing of the optical spectrum of the pulses.

\section{Conclusion}

We have demonstrated a compact integrated microring resonator in SOI material with 0.32nm FSR, an extinction ratio of $27 \mathrm{~dB}$, and a quality factor of $\sim 140900$ around $1550 \mathrm{~nm}$. To our knowledge this is the first experimental demonstration of an SOI microring used as an inherently stable passive optical pulse multiplier. We have demonstrated the generation of a $40 \mathrm{GHz}$ pulse train from a $10 \mathrm{GHz}$ input using this device, and we believe that the integrated microring resonator will be an important device in future high-speed optical communication systems.

\section{Reference}

[1] K.J. Vahala, "Optical microcavities", Nature 424, 839 (2003).

[2] B. Xia and L. R. Chen, "A direct temporal domain approach for pulse-repetition rate multiplication with arbitrary envelope shaping", Selected Topics in Quantum Electronics, IEEE Journal of 11, 165-172 (2005).

[3] M. A. Preciado and M. A. Muriel, "Repetition-rate multiplication using a single all-pass optical cavity", Opt. Lett. 33, $962-964$ (2008).

[4] K.Yiannopoulos, K. Vyrsokinos, E. kehayas, N. Pleros, K. Vlachos, H. Avramopoulos and G. Guekos, "Rate Multiplication by DoublePassing Fabry-Perot Filtering", Photonics Technology Letters, IEEE 15, 1294-1296 (2003).

[5] P. Petropoulos, M.Ibsen, M.N.Zervas, and D.J. Richardson, "Generation of a 40-GHz pulse stream by pulse multiplication with a sampled fiber Bragg grating," Opt. Lett. 25, 521-523 (2000). 